\title{
Patient satisfaction following transition from the original to the new formulation of subcutaneous interferon beta- $l a$ in relapsing multiple sclerosis: a randomized, two-arm, open-label, Phase Illb study
}

\author{
This article was published in the following Dove Press journal: \\ Patient Preference and Adherence \\ 5 May 2010 \\ Number of times this article has been viewed
}

\author{
William Camu' \\ Karim Hadjout ${ }^{2}$ \\ Sabine Latour ${ }^{3}$ \\ Dieter Pöhlau ${ }^{4}$ \\ Said Masri ${ }^{5}$ \\ 'MS Clinic, CHU Gui de Chauliac, \\ University of Montpellier I, \\ Montpellier, France; ${ }^{2}$ Department of \\ Neurology, Centre Hospitalier, Rodez, \\ France; ${ }^{3}$ Merck Serono S.A. - Geneva, \\ Switzerland, an affiliate of Merck \\ KGaA, Darmstadt, Germany; ${ }^{4}$ Multiple \\ Sclerosis Center, Kamillus-Klinik \\ Asbach, Asbach, Germany; ${ }^{5}$ Praxis \\ Masri, Berlin, Germany
}

Correspondence: William Camu MS Clinic, CHU Gui de Chauliac, University of Montpellier I, 2 avenue Bertin-Sans,

34295 Montpellier, France

Tel +33-4-67-33-78-22

Fax +33-4-67-33-8I-36

Email w-camu@chu-montpellier.fr
Objective: To assess satisfaction with the serum-free formulation of subcutaneous (sc) interferon (IFN) beta-1a among patients with relapsing multiple sclerosis (MS).

Methods: Patients with relapsing MS who had been receiving sc IFN beta-1a for at least 6 months, were transitioned to the new formulation, $44 \mu \mathrm{g}$ three times weekly. Patients were randomized to preventative ibuprofen ( $400 \mathrm{mg} 30-60$ minutes prior to injection) or ibuprofen as needed (PRN) for 4 weeks. The primary endpoint was the 'flu-like' symptom (FLS) domain score of the validated Multiple Sclerosis Treatment Concern Questionnaire (MSTCQ).

Results: Of the 117 patients enrolled, 109 (93.2\%) completed the study. Neither group's MSTCQ FLS score showed a clinically meaningful change from baseline to week 4: mean \pm SD changes were $-1.1 \pm 4.4$ in the preventative ibuprofen group and $0.8 \pm 3.6$ in the ibuprofen PRN group. MSTCQ injection system satisfaction and global side-effect scores were unchanged; total and injection-site reaction scores improved moderately in both groups between baseline and week 4 .

Conclusions: Results showed continued or increased levels of satisfaction with the new formulation of sc IFN beta-1a. FLS occurring with the new formulation were generally mild and seldom sufficiently bothersome to require ibuprofen treatment.

Keywords: multiple sclerosis, tolerability, ibuprofen, flu-like symptoms

\section{Background}

Subcutaneous (sc) interferon (IFN) beta-1a (Rebif ${ }^{\circledR}$, Merck Serono S.A. - Geneva, Switzerland, an affiliate of Merck KGaA, Darmstadt, Germany), has been shown in randomized clinical trials to be effective in the treatment of relapsing forms of multiple sclerosis (MS) when administered at doses of 22 or $44 \mu \mathrm{g}$ three times weekly (tiw). ${ }^{1-3}$

The original formulation of sc IFN beta-1 a contains fetal bovine serum (FBS) and human serum albumin (HSA) as excipients. A new formulation of sc IFN beta-1a has been developed that is free from FBS and HSA. This formulation adjustment was made with the intention of reducing immunogenicity and improving the local tolerability of sc IFN beta-1a. These outcomes were assessed in an open-label study in which IFN beta treatment-naïve patients with relapsing MS $(n=260)$ received the new formulation of sc IFN beta-1a, $44 \mu \mathrm{g}$ tiw, for 96 weeks. ${ }^{4}$ This study showed that the new formulation has an improved overall immunogenicity profile compared 
with historical data from patients treated with the original formulation of sc IFN beta-1a in the EVidence of Interferon Dose-response: European North American Comparative Efficacy (EVIDENCE) and Rebif ${ }^{\circledR}$ vs Glatiramer Acetate in Relapsing MS Disease (REGARD) trials. Although the trial of the new formulation was not designed to assess efficacy, relapses remained well controlled over the 96-week study period. Additionally, the proportion of patients experiencing injection-site reactions (ISRs) was lower with the new formulation: ISRs were reported in $30.8 \%$ of patients treated with the new formulation, compared with $85.8 \%$ in the EVIDENCE study and $41.2 \%$ in the REGARD study.,6 'Flu-like' symptoms (FLS) are known to be a common adverse event (AE) of IFN beta, particularly in the period shortly after treatment initiation:3,7-9 In this study, $71.5 \%$ of IFN beta-naïve patients experienced FLS, compared with $49.0 \%$ in the EVIDENCE study and $36.0 \%$ in the REGARD study. Almost all incidences were of mild or moderate severity and transient, and only $8.5 \%$ of patients were experiencing FLS at the 96-week visit. In most patients, FLS can be managed by concomitant use of nonsteroidal anti-inflammatory drugs (NSAIDs) such as ibuprofen; ${ }^{10}$ however, the use of NSAIDs for the management of FLS was permitted but not mandated in this trial. As such, prophylactic medication to prevent FLS was administered by only $38 \%$ of patients on study day 1 , and the authors commented that increasing this proportion may have reduced the incidence of FLS. ${ }^{4}$ Given that FLS are common with IFN-beta treatment and that they can be managed with concomitant NSAID administration, it is worthwhile to investigate how best to use NSAIDs during transition from the previous to the new formulation of sc IFN beta-1a.

Here we present results from the TRANSition From prEvious to new formulation of Rebif ${ }^{\circledR}$ (TRANSFER) study. This was designed to assess patient satisfaction in patients with relapsing MS transitioning from the original to the new formulation of sc IFN beta-1a. An additional objective was to provide physicians with recommendations on how best to manage the transition to the new formulation through the use of concomitant ibuprofen.

\section{Methods}

The TRANSFER study was a randomized, multicenter, two-arm, open-label, phase IIIb study, carried out at 17 centers in France and Germany. The primary objective was to assess patient satisfaction, as measured by the Multiple Sclerosis Treatment Concerns Questionnaire (MSTCQ), ${ }^{11}$ with particular regard to FLS, during the first 4 weeks after transition from the previous formulation of sc IFN beta-1a to the new formulation, in two groups: patients receiving ibuprofen preventatively, and patients receiving ibuprofen PRN. Patients aged 18-60 years with relapsing MS, were eligible for inclusion in the study if they had an Expanded Disability Status Scale score $\leq 5.5$ at entry, and had been administering sc IFN beta-1a for at least 6 months prior to enrollment, using the Rebiject $\mathrm{II}^{\mathrm{TM}}$ (Merck Serono S.A. Geneva, Switzerland) autoinjection device. Exclusion criteria included secondary progressive MS without superimposed relapses (according to the licensed indication of sc IFN beta-1a), the use of any other injectable medications during or after the week prior to screening, the administration of any immunomodulatory MS therapy in addition to sc IFN beta-1a in the 3 months prior to enrollment (combination therapy), a history of chronic pain syndrome, the occurrence of FLS due to any cause in the week prior to baseline, and contraindication to ibuprofen.

The duration of the trial was approximately 10 weeks, including a period of up to 2 weeks between the screening and baseline visits. Four visits were scheduled, beginning with the screening visit (Figure 1). The 4-week treatment period began with the baseline visit, when patients' treatment with the previous formulation of sc IFN beta-1a $44 \mu \mathrm{g}$ was replaced with the new formulation of sc IFN beta-1a $44 \mu \mathrm{g}$ to be taken on the same 3 days each week. Laboratory assessments were performed at the baseline visit. Patients were required to complete a diary card, detailing any FLS, and reporting the use of any concomitant therapy. Diary data were collected between visits 2 and 4. The MSTCQ was self-administered by patients at the baseline visit and again 4 weeks later, at the end of the treatment period, when clinical and laboratory examinations were also performed. The treatment period was followed by a 4-week safety follow-up period, at the end of which a clinical examination and laboratory assessments were performed.

At the baseline visit, patients were randomized in a $1: 1$ ratio to receive either ibuprofen preventatively or ibuprofen PRN, in addition to the new formulation of sc IFN beta-1a. Randomization was performed using sealed envelopes, and the centrally prepared randomization list was kept by an independent clinical research organization. Patients in the preventative ibuprofen (PI) group, were required to take ibuprofen, $400 \mathrm{mg}$ orally, 30-60 minutes prior to each injection of the new formulation of sc IFN beta-1a. They also had the option of taking 2 additional 400-mg doses of ibuprofen at 6-hour intervals if required, within 24 hours of injection. Patients randomized to receive ibuprofen PRN (IPRN group) 


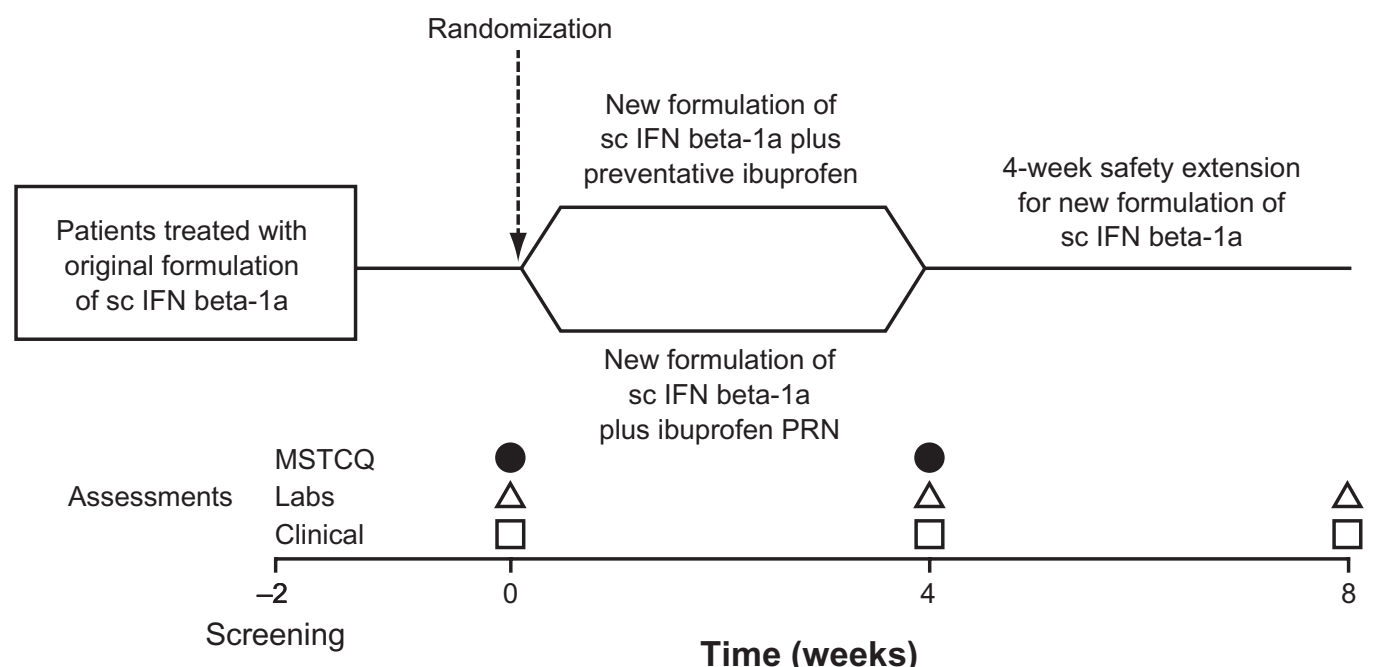

Figure I Study design.

Notes: The original formulation of sc IFN beta- I contains fetal bovine serum (FBS) and human serum albumin (HSA) as excipients. The new formulation of sc IFN beta- Ia is free from FBS and HSA.

Abbreviations: MSTCQ, Multiple Sclerosis Treatment Concerns Questionnaire; PRN, pro re nata (as needed); sc IFN beta- Ia, subcutaneous interferon beta- Ia.

were required not to take ibuprofen before injection, but had the option of taking ibuprofen $400 \mathrm{mg}$ after injection if FLS occurred and were bothersome, with two further 400-mg doses permitted at 6-hour intervals within 24 hours of injection. For both groups, the maximum permitted total dose of ibuprofen per 24 hours was $1200 \mathrm{mg}$.

The primary study endpoint was the score of the FLS component of the MSTCQ at week 4. Secondary endpoints were week 4 scores on the following subscales of the MSTCQ: total score, injection-system satisfaction, ISRs, and global side-effects. Tertiary endpoints were assessments of AEs and serious AEs (SAEs), laboratory assessments (including chemistry, thyroid function, and hematology), physical examination (including vital signs), and documentation of concomitant medications and procedures. The study was conducted in compliance with the ethical principles of the Declaration of Helsinki (1996 amendment) and with European regulatory requirements. All patients were required to provide written informed consent, which was obtained from each patient prior to the conduct of any trial-related procedures not routinely performed as part of the patient's care.

\section{The MSTCQ}

The MSTCQ is a validated 20-item patient questionnaire developed to address patient concerns with IFN-beta treatment that are not related to efficacy. It has two domains: injection-system satisfaction and side effects. The side-effects domain comprises 3 subscales: ISRs, global side effects, and FLS. ${ }^{11}$ All questions in the MSTCQ have a 5 -point response choice, with lower total scores indicating better outcomes.

\section{Analysis populations and statistical methodology}

The intent-to-treat (ITT) population was defined as all patients who underwent randomization. The safety population was defined as all patients receiving $\geq 1$ dose of sc IFN beta- 1 a, and the per-protocol population was defined as all patients in the ITT population, excluding those who had $\geq 1$ major protocol deviation. 'All patients' denotes all patients who provided informed consent. As this study was not hypothesis testing, only descriptive statistics are reported.

Effect sizes were calculated to reflect an estimate of clinically meaningful differences in MSTCQ scores between baseline and week 4 . These effect sizes were calculated as mean change from baseline/standard deviation at baseline. ${ }^{12}$ Effect sizes were classified as follows: a value $\geq 0.2,<0.5$ : small effect; a value $\geq 0.5,<0.8$ : moderate effect; a value $\geq 0.8$ : large effect. ${ }^{11}$ Positive or negative values in effect size indicate only the direction of the change in score. A negative change in MSTCQ score indicates an improvement.

\section{Results}

\section{Patients}

A total of 123 patients gave informed consent; 117 were included in the trial and randomized to the PI $(n=60)$ or the I PRN $(\mathrm{n}=57)$ group. Of the 117 patients enrolled, 
$109(93.2 \%)$ completed the study. Four patients $(6.7 \%)$ in the PI group withdrew prematurely: 3 due to protocol violations and 1 withdrew consent. Four patients $(7.0 \%)$ in the I PRN group withdrew from the study: 1 patient was lost to follow-up, 1 patient withdrew due to AEs (injection-site hematoma), and 2 others withdrew due to 'other' reasons ('severe migraine', and an 'MS attack treated with steroids', respectively).

Patient baseline demographic characteristics were largely similar between the two groups (Table 1), although there was a higher proportion of female patients in the PI group than in the I PRN group ( $80.0 \%$ and $70.2 \%$, respectively). Prior exposure to the previous formulation of sc IFN beta-1a was slightly higher in the I PRN group. Median duration of treatment was 26.5 (range 4-97) months in the PI group and 33.9 (range 4-96) months in the I PRN group. Four patients randomized to the PI group mistakenly took ibuprofen PRN on study day 1 , and another 4 patients randomized to the I PRN group mistakenly took preventative ibuprofen on study day 1 . These 8 patients were therefore included in the safety population according to how they administered ibuprofen treatment on study day 1.

The median number of additional doses of ibuprofen taken during the treatment period was 1 (range 0-31) in the PI group. Patients in the I PRN group took a median of 2 (range 0-24) optional doses of ibuprofen.

\section{MSTCQ results}

There was no clinically meaningful change from baseline to week 4 in the FLS domain score in either treatment arm. The mean total MSTCQ score improved between baseline and week 4 in both treatment groups and the mean scores for ISRs showed an improvement in patients' perception of ISRs from baseline to week 4 in both groups. The mean global side-effects scores showed no worsening in patients' perception of side effects from baseline to week 4 in either group, whereas mean scores in the injection-system satisfaction domain improved from baseline to week 4 in both treatment groups. The mean changes from baseline in the MSTCQ total score and in the individual MSTCQ domains and subscales are shown in Table 2. Where improvements were seen, the effect sizes were small to moderate.

\section{Safety during the 4-week treatment period}

A total of 79/116(68.1\%) patients experienced an AE in at least one of the prespecified AE categories of interest (Table 3). Most AEs were of mild or moderate severity. The AE reported most commonly was FLS: by $66.1 \%$ of patients in the PI group and by $66.7 \%$ of patients in the I PRN group. ISRs were reported in a lower proportion of patients in the I PRN group than in the PI group (3.5\% and 6.8\%, respectively). Table 4 shows the most common AEs during the 4-week treatment period. The safety data show that AEs were consistent with the established safety profile of sc IFN beta-1a. Fatigue, nausea, and injection-site erythema were more common in the PI group than in the IPRN group. Arthralgia, myalgia, migraine, and nasopharyngitis were all more common in the IPRN group than in the PI group. One patient in the IPRN group (1.8\%) withdrew from treatment due to injection-site hematoma, and 1 patient $(1.7 \%)$ in the PI group reported mild abdominal pain as an SAE. This patient was hospitalized for 4 days, after which the pain resolved and study treatment continued uninterrupted. The investigator assessed the event as unlikely to be related to sc IFN beta-1a treatment and indicated that it may have been psychosomatic in origin.

\section{Safety during the 4-week safety extension period}

During the 4-week safety extension period, at least one AE was reported by a total of $73 / 116(62.9 \%)$ patients. A higher

Table I Patient baseline demographics and disease characteristics

\begin{tabular}{|c|c|c|c|c|}
\hline \multicolumn{2}{|l|}{ Patient characteristic } & $\begin{array}{l}\text { Preventative } \\
\text { ibuprofen }(n=60)\end{array}$ & \multirow{2}{*}{$\begin{array}{l}\begin{array}{l}\text { Ibuprofen } \\
\text { PRN (n= 57) }\end{array} \\
41.5 \pm 8.9\end{array}$} & \multirow{2}{*}{$\begin{array}{l}\text { Total }(n=117) \\
41.6 \pm 8.6\end{array}$} \\
\hline Age (years) & Mean \pm SD & $41.7 \pm 8.4$ & & \\
\hline & Median (range) & $42.0(23-60)$ & $43.9(20-58)$ & $43.0(20-60)$ \\
\hline \multirow[t]{2}{*}{ Sex } & Male & $12(20.0 \%)$ & $17(29.8 \%)$ & $29(24.8 \%)$ \\
\hline & Female & $48(80.0)$ & $40(70.2)$ & $88(75.2)$ \\
\hline \multirow[t]{2}{*}{ Number of MS relapses during 24 months } & Mean \pm SD & $1.4 \pm 1.3$ & $1.0 \pm 1.1$ & $1.2 \pm 1.3$ \\
\hline & Median (range) & I.0 (0.0-5.0) & $1.0(0.0-5.0)$ & $1.0(0.0-5.0)$ \\
\hline \multirow[t]{2}{*}{ EDSS score } & Mean \pm SD & $2.3 \pm 1.5$ & $2.0 \pm 1.3$ & $2.2 \pm 1.4$ \\
\hline & Median (range) & $2.0(0.0-5.5)$ & $2.0(0.0-5.5)$ & $2.0(0.0-5.5)$ \\
\hline \multirow[t]{2}{*}{ Exposure to sc IFN beta-I a, months } & Mean \pm SD & $31.0 \pm 23.7$ & $36.8 \pm 26.7$ & $33.8 \pm 25.3$ \\
\hline & Median (range) & $26.5(4.0-97.0)$ & $33.9(4.0-96.0)$ & $29.4(4.0-97.0)$ \\
\hline
\end{tabular}

Abbreviations: EDSS, Expanded Disability Status Scale; IFN, interferon; MS, multiple sclerosis; PRN, pro re nata; sc, subcutaneous; SD, standard deviation. 


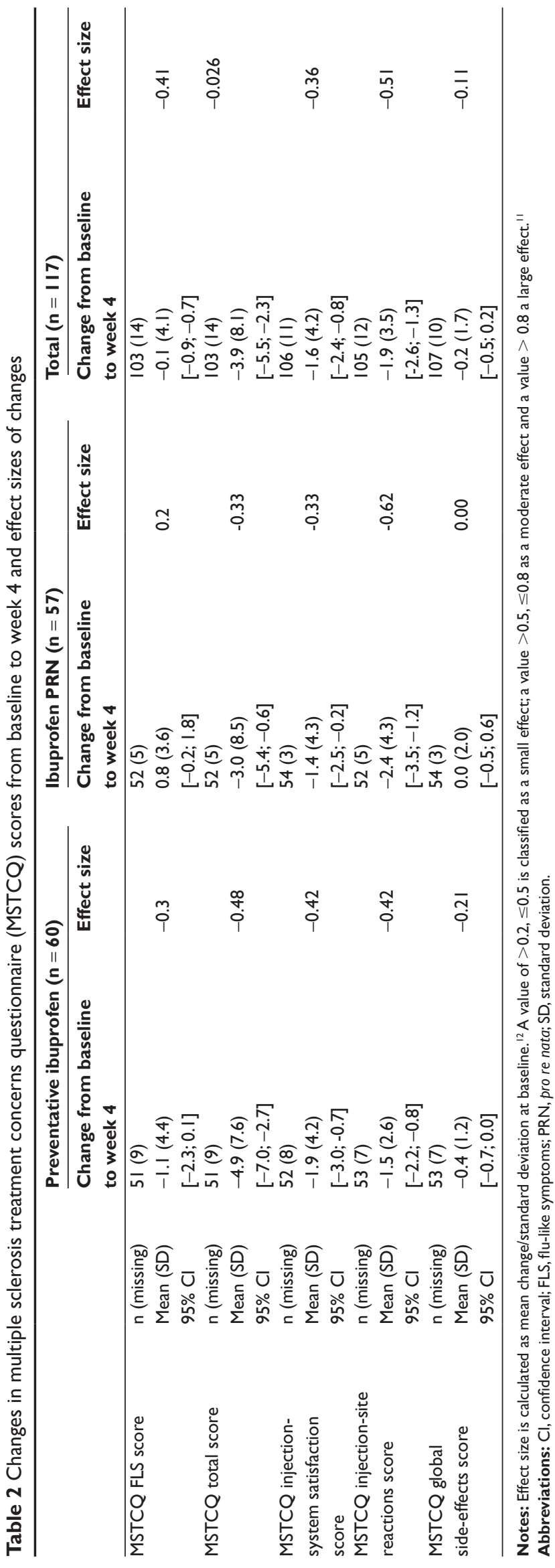

proportion of patients in the PI group (71.2\%) than in the IPRN group (54.4\%) reported AEs. The AEs reported most commonly during this period were FLS, headache, and fatigue, being reported by $46.6 \%, 22.4 \%$, and $12.1 \%$ of all patients, respectively. FLS occurred in a slightly higher proportion of patients in the PI group (52.5\%) than in the IPRN group (40.4\%). No patient experienced an SAE during the safety extension period, and no AEs led to study or IFN beta-1a treatment discontinuation.

\section{Discussion}

This randomized, open-label, phase IIIb study, assessed satisfaction in patients with relapsing MS when transitioning from the original formulation of sc IFN beta-1a to a new formulation produced without FBS and without HSA as excipiens.

There was no clinically meaningful change from baseline in the score for the FLS component of the MSTCQ in either treatment group, indicating that FLS are not an obstacle in the transition to the new formulation of sc IFN beta-1a, and that any FLS that occur during transition can be easily managed with ibuprofen taken either preventatively or PRN. Although FLS were experienced by approximately two-thirds of patients in each group at some point during the study, the optional use of ibuprofen was low in both groups, showing that when FLS appeared, patients seldom considered them sufficiently bothersome to require treatment.

Both treatment groups showed improvements from baseline to week 4 in the mean MSTCQ total score and injection-system satisfaction score, with a small overall effect size in each case, and an improvement in the mean MSTCQ ISR score, with a moderate effect size. There was no change from baseline in the mean MSTCQ global side-effects score in either group. These results indicate that patients transitioning from the previous formulation of sc IFN beta-1a to the new formulation, were either equally or more satisfied with their new treatment.

A lower proportion of patients experienced FLS during the 4-week safety extension period (46.6\%) than during the treatment period $(66.4 \%)$. During the safety extension period, FLS were experienced by a higher proportion of patients randomized to preventative ibuprofen (52.5\%) than those randomized to ibuprofen PRN (40.4\%). However, it is unclear whether this was due to an actual higher incidence rate of FLS in the 'previously preventative' patients following a switch to PRN use of ibuprofen, or whether FLS were perceived more acutely by these patients following the switch in ibuprofen regimen. 
Table 3 Prespecified adverse events during the 4-week treatment period (safety population)

\begin{tabular}{llll}
\hline & \multicolumn{1}{l}{ Treatment } & & \\
\cline { 2 - 4 } & Preventative ibuprofen $(\mathbf{n}=\mathbf{5 9})$ & Ibuprofen PRN $(\mathbf{n}=\mathbf{5 7})$ & $\mathrm{T}$ \\
\hline Patients & $\mathrm{n}(\%)$ & $\mathrm{n}(\%)$ & $\mathrm{n}(\%)$ \\
Adverse event & & & \\
Any prespecified adverse event & $40(67.8)$ & $39(68.4)$ & $79(68.1)$ \\
Depression and suicidal ideation & $0(0.0)$ & $\mathrm{I}(1.8)$ & $\mathrm{I}(0.9)$ \\
Flu-like symptoms & $39(66.1)$ & $38(66.7)$ & $77(66.4)$ \\
Injection-site reactions & $4(6.8)$ & $2(3.5)$ & $6(5.2)$ \\
Skin rashes & $2(3.4)$ & $0(0.0)$ & $2(1.7)$ \\
Thyroid disorders & $2(3.4)$ & $0(0.0)$ & $2(1.7)$ \\
\hline
\end{tabular}

Abbreviation: PRN, pro re nata.

The changes in MSTCQ scores for ISRs and injection system satisfaction following treatment transition indicate improved patient rating of ISRs and the injection process with the new formulation. Indeed, this was one of the key objectives for which the new formulation of sc IFN beta-1a was developed. The improvement in the rating of injection system satisfaction must actually reflect the difference in patient perception due to the change in formulation, because all patients used the same injection device both before and after the transition.

The overall incidence of FLS during the treatment period was higher than in the sc IFN beta-1a arms of the EVIDENCE and REGARD studies of the previous formulation, but lower than that in the open-label, single-arm study of the new formulation of sc IFN beta-1a, which did not mandate ibuprofen treatment. The incidence of ISRs in the present study was considerably lower than those reported for the previous studies mentioned above. However, these inconsistencies are not surprising, as no direct comparison can be made with any of these trials because of differences in study design and duration.

\section{Conclusions}

Overall in this study, patient ratings of ISRs improved and patients reported either equal or greater levels of satisfaction with the new formulation of IFN beta-1a compared with the original formulation. As these patients had received stable treatment with the previous formulation for at least 6 months

Table 4 Most common adverse events during the 4-week treatment period*

\begin{tabular}{|c|c|c|c|c|c|c|}
\hline \multirow[t]{4}{*}{ Adverse event } & \multicolumn{6}{|l|}{ Treatment } \\
\hline & \multicolumn{2}{|c|}{ Preventative ibuprofen } & \multicolumn{2}{|l|}{ Ibuprofen PRN } & \multicolumn{2}{|l|}{ Total } \\
\hline & $\begin{array}{l}\text { Patients } \\
(n=59)\end{array}$ & $\begin{array}{l}\text { Events } \\
(n=3 \mid 3)\end{array}$ & $\begin{array}{l}\text { Patients } \\
(n=57)\end{array}$ & $\begin{array}{l}\text { Events } \\
(n=272)\end{array}$ & $\begin{array}{l}\text { Patients } \\
(n=116)\end{array}$ & Events $(n=585)$ \\
\hline & Patients, n (\%) & Events, n (\%) & Patients, n (\%) & Events, n (\%) & Patients, n (\%) & Events, n (\%) \\
\hline Flu-like symptoms & $39(66.1)$ & $182(58.1)$ & $38(66.7)$ & $170(62.5)$ & $77(66.4)$ & $352(60.2)$ \\
\hline Headache & $22(37.3)$ & $41(13.1)$ & $16(28.1)$ & $33(12.1)$ & $38(32.8)$ & $74(12.6)$ \\
\hline Fatigue & $15(25.4)$ & $36(1 \mathrm{I} .5)$ & $7(12.3)$ & $10(3.7)$ & $22(19.0)$ & $46(7.9)$ \\
\hline Myalgia & $3(5.1)$ & $3(1.0)$ & $5(8.8)$ & $17(6.3)$ & $8(6.9)$ & $20(3.4)$ \\
\hline Chills & $4(6.8)$ & $5(1.6)$ & $4(7.0)$ & $5(1.8)$ & $8(6.9)$ & $10(1.7)$ \\
\hline Arthralgia & $2(3.4)$ & $2(0.6)$ & $4(7.0)$ & $10(3.7)$ & $6(5.2)$ & $12(2.1)$ \\
\hline Back pain & $3(5.1)$ & $3(1.0)$ & $2(3.5)$ & $2(0.7)$ & $5(4.3)$ & $5(0.9)$ \\
\hline Migraine & $0(0.0)$ & $0(0.0)$ & $4(7.0)$ & $5(1.8)$ & $4(3.4)$ & $5(0.9)$ \\
\hline Nasopharyngitis & $2(3.4)$ & $2(0.6)$ & $3(5.3)$ & $3(1.1)$ & $5(4.3)$ & $5(0.9)$ \\
\hline Pyrexia & $2(3.4)$ & $4(1.3)$ & $\mathrm{I}(\mathrm{I} .8)$ & I $(0.4)$ & $3(2.6)$ & $5(0.9)$ \\
\hline Vertigo & $0(0.0)$ & $0(0.0)$ & $3(5.3)$ & $3(I . I)$ & $3(2.6)$ & $3(0.5)$ \\
\hline Injection-site pain & I (I.7) & $2(0.6)$ & $\mathrm{I}(\mathrm{I} .8)$ & $\mathrm{I}(0.4)$ & $2(1.7)$ & $3(0.5)$ \\
\hline Abdominal pain & I (I.7) & $\mathrm{I}(0.3)$ & $\mathrm{I}(\mathrm{I} .8)$ & I (0.4) & $2(1.7)$ & $2(0.3)$ \\
\hline $\begin{array}{l}\text { Antithyroid antibody } \\
\text { positive }\end{array}$ & $2(3.4)$ & $2(0.6)$ & $0(0.0)$ & $0(0.0)$ & $2(1.7)$ & $2(0.3)$ \\
\hline Diarrhea & I (I.7) & $\mathrm{I}(0.3)$ & $\mathrm{I}(\mathrm{I} .8)$ & I (0.4) & $2(1.7)$ & $2(0.3)$ \\
\hline Injection-site erythema & $2(3.4)$ & $2(0.6)$ & $0(0.0)$ & $0(0.0)$ & $2(1.7)$ & $2(0.3)$ \\
\hline Nausea & $2(3.4)$ & $2(0.6)$ & $0(0.0)$ & $0(0.0)$ & $2(1.7)$ & $2(0.3)$ \\
\hline
\end{tabular}

*Occurring in $\geq 1 \%$ of patients. PRN, pro re nata. 
(and the majority for more than 2 years), a reasonable level of treatment satisfaction with the previous formulation is implicit. Patient satisfaction with treatment is important in the period following a therapy adjustment, when patients may be at heightened risk of discontinuation. In addition, the transition to the new formulation was well tolerated and FLS, when they occurred, were mild and rarely considered to be sufficiently bothersome to warrant treatment. However, if required, such symptoms were easily managed with ibuprofen, administered either preventatively or PRN.

\section{Acknowledgments}

The authors take full responsibility for the content of the paper but thank Steve Smith, Caudex Medical (supported by Merck Serono S.A. - Geneva, Switzerland, an affiliate of Merck KGaA, Darmstadt, Germany), and Sarah-Jane Loveday (Merck Serono S.A. - Geneva, Switzerland) for assistance in the preparation of the manuscript.

\section{Disclosure}

William Camu has received consulting fees from Merck Serono, TEVA Pharma, and Sanofi-Aventis, and research grants from Bayer Schering, Biogen Idec, Merck Serono, TEVA Pharma, and Sanofi-Aventis. Sabine Latour is a salaried employee of Merck Serono S.A. - Geneva. Dieter Pöhlau has received speaker fees from Bayer Schering Pharma, Teva, Aventis, and Biogen Idec. Karim Hadjout and Said Masri have no conflicts of interest to declare.

\section{References}

1. Kappos L, Traboulsee A, Constantinescu C, et al. Long-term subcutaneous interferon beta-1a therapy in patients with relapsing-remitting MS. Neurology. 2006;67(6):944-953.

2. PRISMS (Prevention of Relapses and Disability by Interferon beta-1a Subcutaneously in Multiple Sclerosis) Study Group; and the University of British Columbia MS/MRI Analysis Group. PRISMS-4: long-term efficacy of interferon beta-1a in relapsing MS [published correction appears in Neurology. 2001;25;57(6):1146]. Neurology. 2001;56(12):16281636.
3. PRISMS (Prevention of Relapses and Disability by Interferon beta-1a Subcutaneously in Multiple Sclerosis) Study Group. Randomised double-blind placebo-controlled study of interferon beta1a in relapsing/remitting multiple sclerosis [published correction appears in Lancet. 1999;353(9153):678]. Lancet. 1998;352(9139): 1498-1504.

4. Giovannoni G, Barbarash O, Casset-Semanaz F, et al. Safety and immunogenicity of a new formulation of interferon beta-1a (Rebif New Formulation) in a Phase IIIb study in patients with relapsing multiple sclerosis: 96-week results. Mult Scler. 2009;15(2): 219-228.

5. Mikol DD, Barkhof F, Chang P, et al. Comparison of subcutaneous interferon beta-1a with glatiramer acetate in patients with relapsing multiple sclerosis (the REbif vs Glatiramer Acetate in Relapsing MS Disease [REGARD] study): a multicentre, randomised, parallel, open-label trial. Lancet Neurol. 2008;7(10): 903-914.

6. Panitch H, Goodin DS, Francis G, et al. EVidence of Interferon Dose-response: European North American Comparative Efficacy; University of British Columbia MS/MRI Research Group. Randomized, comparative study of interferon beta-1a treatment regimens in MS: the EVIDENCE Trial. Neurology. 2002;59(10):1496-1506.

7. Jacobs LD, Cookfair DL, Rudick RA, et al. Intramuscular interferon beta-1a for disease progression in relapsing multiple sclerosis. The Multiple Sclerosis Collaborative Research Group (MSCRG) [published correction appears in Ann Neurol. 1996;40(3):480]. Ann Neurol. 1996;39(3):285-294.

8. The IFNB Multiple Sclerosis Study Group. Interferon beta-1b is effective in relapsing-remitting multiple sclerosis. I. Clinical results of a multicenter, randomized, double-blind, placebo-controlled trial. Neurology. 1993;43(4):655-661.

9. Bayas A, Rieckmann P. Managing the adverse effects of interferon-beta therapy in multiple sclerosis. Drug Saf. 2000;22(2):149-159.

10. Río J, Nos C, Bonaventura I, et al. Corticosteroids, ibuprofen, and acetaminophen for IFNbeta-1a flu symptoms in MS: a randomized trial. Neurology. 2004;63(3):525-528.

11. Cramer JA, Cuffel BJ, Divan V, Al-Sabbagh A, Glassman M. Patient satisfaction with an injection device for multiple sclerosis treatment. Acta Neurol Scand. 2006;113(3):156-162.

12. Kazis LE, Anderson JJ, Meenan RF. Effect sizes for interpreting changes in health status. Med Care. 1989;27(3 Supp1):S178S189.
Patient Preference and Adherence

\section{Publish your work in this journal}

Patient Preference and Adherence is an international, peer-reviewed, open access journal that focusing on the growing importance of patient preference and adherence throughout the therapeutic continuum. Patient satisfaction, acceptability, quality of life, compliance, persistence and their role in developing new therapeutic modalities and compounds to

\section{Dovepress}

optimize clinical outcomes for existing disease states are major areas of interest. This journal has been accepted for indexing on PubMed Central. The manuscript management system is completely online and includes a very quick and fair peer-review system. Visit http://www.dovepress.com/ testimonials.php to read real quotes from published authors. 